https://doi.org/10.33996/reba.vli2.4

\title{
Procesos de negocio de la banca universal en la Costa Oriental del Lago
}

\section{Business plans of the universal banking on the East Coast of Lake}

\author{
4 Isabelina Gutiérrez \\ isabelgps@hotmail.com \\ ORCID: 0000-0003-0476-6874 \\ Banco Occidental de Descuento \\ Artículo recibido en enero 2019 / Arbitrado en febrero 2019 / Publicado en julio 2019
}

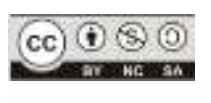

RESUMEN

La presente investigación tuvo como objetivo describir los procesos de negocio de la banca universal en Costa Oriental del Lago. El tipo de investigación fue descriptiva bajo un diseño no experimental, de campo, transaccional. La población quedó conformada por 64 unidades informantes, integrada por gerentes, subgerentes, asesores, ejecutivos y gerentes de zonas, de 14 bancos activos al momento de la investigación. El instrumento utilizado fue el cuestionario con un total de 14 ítems. La validación estuvo a cargo por el juicio de expertos y su confiabilidad a través del método de Alpha de Cronbach, cuyo resultado fue 0,98. El análisis de los datos se realizó a través de la estadística descriptiva, por medio de la media aritmética. Se concluyó que los bancos objeto de estudio siempre utilizan la base del negocio, el área del negocio y las operaciones del negocio como procesos de negocio que manejan recursos financieros.

Palabras clave:Área del negocio, banca universal, base del negocio, operaciones del negocio, procesos de negocio, recursos financieros

ABSTRACT

The objective of this research was to describe the business processes of universal banking in the Eastern Coast of the Lake. The type of research was descriptive under a non-experimental, field, transactional design. The population was made up of 64 reporting units, made up of managers, assistant managers, advisers, executives and area managers, from 14 banks active at the time of the investigation. The instrument used was the questionnaire with a total of 14 items. Validation was carried out by expert judgment and its reliability through Cronbach's Alpha method, whose result was 0.98. The data analysis was performed through descriptive statistics, using the arithmetic mean. It was concluded that the banks under study always use the business base, the business area and the business operations as business processes that manage financial resources.

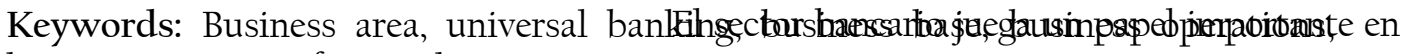
business processes, financial resourcesel crecimiento de la economía, debido a que sirve de

INTRODUCCIÓN estímulo para el desarrollo de diversas actividades que constituye el motor principal de la misma. Esto es así, 
porque la banca cumple dos funciones de primordial importancia, como lo son: la prestación de un servicio de sistema de pago que facilita las transacciones entre los agentes y la intermediación financiera en la que lograr captar y aprovechar los flujos de dinero de aquellos individuos que tienen la capacidad de ahorro, para destinarlos hacia otros sectores.

En este contexto, el proceso de negocio, es vital como herramienta para alcanzar la rentabilidad de las entidades financieras. Estas entidades financieras existen para atender las necesidades financieras de sus clientes y del público en general. Igualmente, persiguen una utilidad para retribuir la inversión de sus accionistas.

$\mathrm{Al}$ respecto, Santos (2001), refiere que el negocio bancario se realiza a través de los Bancos, al ser estos intermediarios financieros, reciben el dinero del público y lo colocan en el público. Por este servicio obtienen un margen de intermediación (diferencia entre el interés que pagan por el ahorro y el que cobran por los créditos), y adicionalmente cobran por los servicios prestados (comisiones, cuotas de manejo).

Sin embargo, se observa como la banca universal utiliza sistemas de mediciones para observar primordialmente el desarrollo de sus operaciones, siendo necesario aplicar una serie de procesos de negocio como instrumentos para crecer en el mercado y prever el logro de niveles de competitividad, más aun teniendo en cuenta que la banca universal a través de la intermediación financiera, presta un servicio orientado a cubrir múltiples necesidades de distintos usuarios.

Específicamente, la Costa Oriental del Lago, actualmente consta de 14 bancos universales, que en su búsqueda de estrategias que permitan gestionar el mercado financiero para transitar exitosamente el sinuoso camino que se presenta en el dificultoso terreno financiero, hace uso de los procesos de negocio, con el fin, de poner en acción en forma apropiada las estrategias de negocios, tomando en consideración los riesgos que debe el banquero asumir desde la intermediación de baja contingencia y escaso dinamismo económico, hasta la financiación de innovaciones que se asocian a la más alta exposición. Bajo esta óptica, en el presente artículo, se describen los procesos de negocio de la banca universal en Costa Oriental del Lago.

En primer lugar, se define los procesos de negocio, para Sommerville (2005) son los procesos utilizados en los negocios para lograr algún objetivo del mismo. Según el citado autor, estos pueden ser diseñados alrededor de un sistema heredado y restringido por la funcionalidad que este proporciona.

Asimismo, para Laudon (2004), los procesos de negocios se refieren a la manera especial de organizar, coordinar y enfocar el trabajo para elaborar un producto o servicio valioso. Desarrollar un producto nuevo, generar y completar un pedido o contratar un empleado de negocios puede ser una fuente de fortaleza competitiva

Según, De Carlos (2004), el proceso de negocio , también es conocido como naturaleza de las organizaciones, modelo muy actual y que recién se está incorporando en forma sistemática al mundo empresarial y directivo para enriquecer el análisis estratégico, consiste en la identificación del conjunto de orientaciones, funciones y actividades que tienen directa relación y de los que dependen en forma decisiva, y principal, los resultados económicos en términos de ingresos (mayores o menores) o las pérdidas o ganancias o el cumplimiento de sus propósitos esenciales.

Refiere el autor, que el proceso de negocio es una parte del proceso de la naturaleza de la organización, ya que este último hace referencia a los resultados no sólo económicos, sino al cumplimiento de los objetivos que por naturaleza y esenciales características corresponden a la organización. Mientras el proceso de la naturaleza es propio de toda organización (familia, región, organizaciones con o sin fin de lucro, privados, estatales mixtos, etc.), el proceso de negocio, una especialificidad dentro de aquél, agrega el aspecto financiero de ingresos, pérdidas o ganancias, en las organizaciones en las que esto es requerido. 
Ahora bien, en principio, casi todas las actividades que se realizan en las empresas $u$ organizaciones tienen algo que ver con sus resultados económicos o tienen repercusiones económicas, pero no todas son igualmente determinantes del logro de dichos resultados. Hay así una jerarquización de la mayor dependencia de los resultados de unos u otras orientaciones, funciones o actividades, si bien todas ellas se realizan en las empresas.

Se trata entonces, de descubrir, describir y clasificar prácticamente en qué orientaciones, funciones y actividades de la organización están en juego los ingresos y retornos de la empresa de manera más destacada (de qué dependen los resultados en beneficios o pérdidas: proceso de negocio); y/o de qué depende cumplir o no con los objetivos y propósitos de la naturaleza que tipifican a la tal organización (proceso de la naturaleza, ampliable a instituciones aun sin fines de lucro sea para particulares o para el Estado).

Desde estas perspectivas, a criterio de la investigadora, el proceso de negocio, es una serie de pasos realizados por la banca universal en busca de mejorar la eficiencia y la eficacia por medio de la gestión sistemática de los mismos. Dichos pasos tienen un orden específico de actividades de trabajo, que se realizan en el tiempo, en lugares específicos y por personas o sistemas, con un comienzo, un fin, con entradas y salidas claramente definidas. Es decir, una estructura cohesionada coordinada adecuadamente para la acción que consta de la bases del negocio, áreas del negocio y las operaciones del negocio.

En segundo lugar, se encuentran las bases del negocio que para Charan (2007) tienen su idea central en el posicionamiento sobre el cual se gana o no el dinero, si a las personas les gusta lo que tienen para ofrecerles y se puede vender y derivar de la venta una ganancia, entonces se hará dinero pero si están confundidos acerca de lo que el negocio proporciona o no les gusta no habrán ganancias y para mantenerse en el negocio para que encaje en el panorama cambiante, tendrán que reposicionar su negocio. Esto significa tomar decisiones básicas sobre que adicionarles y que prescindir, identificar nuevas oportunidades de crecer, segmentar de otras formas los mercados y decidir sobre cuáles son las nuevas tecnologías que deben adoptar.

Por su parte, De Carlos (2004), plantea que las bases del negocio son los rasgos distintivos, identificatorios, claves y originales que hacen a una empresa u organización lo que es (su naturaleza) y la definen desde el punto de vista de sus resultados "económicos y/o típicos o propios de su quehacer.

Su identificación y definición en la organización constituye el primer paso para el análisis del proceso de negocio, o típico, o de la naturaleza de la organización. La definición del negocio es conveniente que se haga en forma precisa y clara. Consiste en matices identificatorios que describan por qué y cómo se espera conseguir los resultados generales y, sobre todo, económicos.

Las bases del negocio descansan, para su descubrimiento por parte del directivo y para su posterior relación y formulación, en la definición de unos rasgos distintivo, diferenciadores que singularicen la empresa y que se refieran a los ámbitos productivos, tecnología, mercado, financiamiento, personal, ámbito geográfico, formas y orientaciones de distribución. Manifestando unas "ventajas competitivas" apoyadas en la realidad de los antecedentes observables en el sector y propia empresa u organización (la base sería "buscar la diferencia ventajosa” en la generación de resultados). Superar a los competidores de algún modo novedoso y original, observar carencias o necesidades no atendidas (anticipación).

Esto requiere que tales ventajas "sean sostenibles" en el tiempo, es decir, no fácilmente duplicables por el resto de las empresas del sector 13 ellas pueden significar primacías en costos, variedad de productos, facilidades de pago para adquisición, nuevos procesos productivos o de distribución, terminaciones y calidad al lado de un precio, vanguardia tecnológica, segmento definido de mercado.

Para Abadal (2001), las bases del negocio contienen información económica, comercial, en negocios dirigidas principalmente a las empresas en especial a las tienen importantes volúmenes de facturación y departamentos financieros se pueden 
distinguir dos tipos de orientación: información financiera y el análisis e investigación de mercados.

La información financiera se trata de bases de datos que se ocupan de registrar valores de fluctuación constante como índices de bolsas de mercado de futuras divisas entre otras. El análisis e investigación de mercado tienen informaciones de negocio útiles para la empresa, créditos, directorios económicos y empresariales, memorias y balances de empresas. Los servicios en línea de información económica es una de las partes más importantes del negocio generado por las bases.

A los efectos de esta investigación, la base del negocio bancario está en las relaciones estables y directas con los clientes. Un modelo de negocio recurrente, bien gestionado y basado en la relación con el cliente a largo plazo propicia la rentabilidad de las entidades financieras y a los empresarios les aporta valor, servicios y productos ajustados a sus necesidades.

En tercer lugar, se definen las aéreas de negocio, según Casado y Sellers (2006) se conocen como unidades estratégicas del negocio, pueden ser el departamento de una empresa o una línea de productos que tienen una cartera de negocios con la necesidad de un crecimiento rentable para la empresa, valorar y seleccionar oportunidades de mercado y elaborar estrategias para alcanzarlas con su objetivo correspondiente.

En el mismo orden de idas, para Muñiz (2003), área de negocio es una unidad organizativa que asume la responsabilidad de realizar todas las funciones y trabajo ya que tienen capacidad de decisión y una visión global del funcionamiento de su unidad. La estructura por áreas de negocio es más efectiva cuando los costos por departamento centralizados son muy reducidos.

Acota, De Carlos (2004), que las áreas de negocio son segmentos distintivos e identificables del entorno en el cual la empresa hace (actuales áreas) o puede hacer (áreas potenciales), o sea, generar ingresos o beneficios o identificar actividades, gravámenes o restricciones a éstos. Se identifica cada área de negocio por relaciones de demanda y oferta, producto, mercado, tecnología que se dirige al sector (demanda) y/o se entregan por éste o por algunas empresas integrantes del mismo.

En lo que refiere los mercados altos, medios, bajos, intermedios segmentos, y respecto de cada país el significado de producto de calidad alta, media, baja tiene diferentes sentidos y precios y tratamientos legales o canales de distribución. Ella da origen a múltiples áreas de negocio. Asimismo, la tecnología sea por razones de características de los insumos o de los componentes empleados, los procesos de fabricación, transporte, almacenaje, carga, descarga, packing, recepción, entre otros., van dando lugar a otras tantas posibilidades de área de negocio.

Las diferencias, sea en producto, mercados, o tecnología, van a dar origen a las áreas de negocio en que la empresa se desenvuelve (actuales áreas), siendo el resto áreas potenciales de acción que requeriría para poder incorporarlos al negocio. Obviamente que en otras organizaciones habrá áreas de la naturaleza o típicas de cumplimiento de su misión o esencia. Un portafolio o combinado de áreas permitiría mejorar la posición de la compañía, un estudio detallado de ellas, anticipar el comportamiento de cada una, su evolución y la estrategia a seguir respecto del cultivo de las áreas actuales, y el desarrollo contenido, movimiento y acciones, para hacer aparecer nuevas áreas en mi empresa (diagnóstico de cada área de negocio actual y prospección de los potenciales).

Desde estas consideraciones, a juicio de la investigadora, las áreas de negocio, son segmentos identificables del entorno con el que la banca hace o puede hacer negocio, a fin de responder con su oferta a unas demandas de producto, mercado-clientes, tecnología, geografía diferenciados, actuando competitivamente buscando maximizar ingresosfacturación y resultados-beneficios.

En cuaro lugar, continuando con el tema es importante conocer las operaciones de negocios, para Estupiñan (2006), deben revelar si tienen mecanismo de seguridad óptimos en la negociación, si se hicieron en las condiciones pactadas propias de la entidad que aseguren la comprobación de las condiciones pactadas para evitar suspicacias en el momento de la verificación de las operaciones, indicando además si la contabilización de las operaciones se realiza rápida y 
precisa para evitar errores que puedan significar perdidas.

Asimismo, De Carlos (2004), refiere que estas son las actividades o elementos identificables en cada área de negocio y de las que dependen y tiene mayor impacto relativo en el logro de los resultados de dicha área de negocios. Cada área de negocios tiene sus propias operaciones de las que depende obtener resultados económicos (o extraeconómicos: Logro): depende del desarrollo de actividades diversas.

Se trata de examinar cada área de negocios para ver qué actividades, funciones, tareas hay que realizar para que esa área específica genere ingresos o aportaciones a los resultados. Descubrir los componentes operativos de ella, y que suelen ser concreciones específicas de las funciones típicas de la empresa: diseñar, comprar, producir, verificar calidad, vender, promocionar, distribuir, financiar, cobrar, pagar, trabajar con personas.

Identificar, desglosar, describir, calcular, en síntesis, conocer las 15 ó 20 operaciones de las que depende cada área de negocio clave de la organización, su descripción cualitativa y la cuantificación relativa de su incidencia económica y el aporte a los beneficios, o al logro de resultados (en organizaciones sin fin de lucro) que va a permitir dirigir mejor el negocio.

Reseña el citado autor, que las operaciones de negocio dependen entre otras de: a) la situación actual de la organización, su fortaleza, debilidades, posibilidades, incapacidades o capacidades; b) de la evolución de las respuestas que otras organizaciones están dando y de éxito o fracaso con que las están realizando (búsqueda de diferenciación aporte nuevo o condiciones severas), c) de la evolución del entorno sectorial (clientes, intermediarios, financistas, disponibilidades económicas, apetencias.) y macroentorno.

Por ello, las operaciones de negocio de las que depende el desarrollo de un área (SBA) son cambiantes, y las que fueron identificadas como apropiadas en un escenario, no necesariamente continuarán siéndolo en otros y ante otros requerimientos.
A criterio de la investigadora, las operaciones de negocio, es todo lo que pasa dentro de la banca para mantenerla de pie y ganando dinero. Estas varían según el tipo de negocio, industria y tamaño, las mismas deben tomar en consideración algunos elementos, tales como: proceso, personal, ubicación, equipo y tecnología.

De igual manera, considera la investigadora que, una vez que están establecidas las operaciones de negocio y particularmente después del crecimiento, es importante evaluar y analizar periódicamente dichas operaciones para identificar ineficiencias y mejorar la comunicación.

\section{MATERIALES Y MÉTODO}

La investigación por sus características metodológicas se ubicó como descriptiva, bajo un diseño no experimental, puesto que su fin es la observación de la variable y no la manipulación de ésta, de la misma manera, la investigación se enmarca dentro de la tipología de campo y transeccional, ya que se estudia y se describen los datos obtenidos en forma directa de la realidad y en un periodo de tiempo determinado, sin ningún tipo de interrupciones.

La población quedó conformada por 64 unidades informantes, integrada por gerentes, subgerentes, asesores, ejecutivos y gerentes de zonas, de 14 bancos activos al momento de la investigación. El instrumento utilizado fue el cuestionario con un total de 14 ítems, con opciones de respuestas tipo selección múltiple, que consta de cinco (5) alternativas de respuesta: totalmente de acuerdo (5), medianamente de acuerdo (4), ni de acuerdo ni en desacuerdo (3), medianamente en desacuerdo (2), totalmente en desacuerdo (1).

La validación estuvo a cargo por el juicio de expertos y su confiabilidad a través del método de Alpha de Cronbach, cuyo resultado fue 0,98. El análisis de los datos se realizó a través de la estadística descriptiva, por medio de la media aritmética, cuyos valores permitieron describir los procesos de negocio, tal como se muestra en el cuadro 1 . 
Cuadro l. Categoría de análisis para la interpretación del promedio

\begin{tabular}{crll}
\hline Rango & Intervalo & Categoría & \multicolumn{1}{c}{ Descripción } \\
\hline 5 & $4.21-5.00$ & Siempre & $\begin{array}{l}\text { Indica una frecuencia muy alta de la actividad o } \\
\text { proceso analizado. }\end{array}$ \\
4 & $3.41-4.20$ & Casi siempre & $\begin{array}{l}\text { Indica una alta frecuencia de la actividad o proceso } \\
\text { analizado. }\end{array}$ \\
3 & $2.61-3.40$ & $\begin{array}{l}\text { Algunas } \\
\text { Veces }\end{array}$ & $\begin{array}{l}\text { Indica una media frecuencia de la actividad o proceso } \\
\text { analizado. } \\
\text { Indica una baja frecuencia de la actividad o proceso } \\
\text { analizado. } \\
\text { Indica una frecuencia muy baja de la actividad o } \\
\text { proceso analizado. }\end{array}$ \\
\hline
\end{tabular}

Fuente: La investigadora (2020)

\section{RESULTADOS Y DISCUSION}

Como puede observarse en la tabla 1 , se evidencia los resultados para el indicador bases del negocio, el cual alcanzo un promedio de 4,65 ubicándose en la categoría siempre lo que indica que las personas encuestadas tienen dominio sobre las bases de negocios establecidas por los bancos universales orientadas hacia los ámbitos productivos, tecnológicos y de mercados. Al detalle se observa, que la banca analizada siempre establece dentro de sus bases de negocios los rasgos distintivos del producto, al tiempo que buscan diferencia ventajosa en la generación de resultados, de manera que están orientadas a los ámbitos productivos, tecnológicos, dentro del mercado con el financiamiento adecuado.

Los resultados alcanzados, a juicio de la investigadora, permiten afirmar que las bases del negocio siempre forman parte de los procesos de negocio de la banca universal en Costa Oriental del Lago, así las cosas, quienes gestionan estos procesos toman decisiones básicas al identificar nuevas oportunidades de crecer a fin de segmentar otras formas de mercados y decidir sobre cuáles son las nuevas tecnologías que deben adoptar.

Tabla 1. Indicador: Bases del negocio

\begin{tabular}{llcc}
\hline \multicolumn{1}{c}{ ÍTEM } & $\overline{\mathbf{X}}$ & CATEGORÍA \\
\hline 1. & $\begin{array}{l}\text { La organización establece dentro de sus bases de negocios } \\
\text { los rasgos distintivos del producto. }\end{array}$ & 4,76 & Siempre \\
2. $\quad \begin{array}{l}\text { Las bases del negocio establecido por el banco buscan } \\
\text { diferencia ventajosa en la generación de resultados. }\end{array}$ & 4,46 & Siempre \\
3. & Las bases del negocio están orientadas a los ámbitos \\
productivos. & 4,72 & Siempre \\
4. & & 4,57 & Siempre \\
negocio ámbitos tecnológicos están orientados en las bases del & 4,78 & Siempre \\
6. & Las bases del negocio se orientan al mercado & 4,60 & SIEMPRE \\
\hline
\end{tabular}

Fuente: La investigadora (2020) 
Esta situación mostrada, pone en evidencia muy alta coincidencia con De Carlos (2004), para quien las bases del negocio representan los rasgos claves y originales que hacen a una empresa y la definen desde el punto de vista de sus resultados económicos y/o típicos o propios de su quehacer. Asimismo, coincide con lo expuesto por Charan (2007) quien manifiesta que las bases del negocio tienen su idea central en el posicionamiento sobre el cual se gana o no el dinero, significa tomar decisiones básicas e identificar nuevas oportunidades de crecer.

Con base a lo descrito, puede decirse entonces que la banca universal estudiada, se maneja un modelo de negocio recurrente, bien gestionado y basado en la relación con el cliente a largo plazo propicia la rentabilidad de las entidades financieras y a los empresarios les aporta valor, servicios y productos ajustados a sus necesidades, validando con ello el criterio de la investigadora.
En lo concerniente al indicador áreas de negocio, se observa, en la tabla 2, un promedio de 4,62, ubicándola en la categoría siempre, al considerar los encuestados que el personal está bien claro en que la banca universal a través de sus operaciones de negocio pueden generar un gran mercado competitivo apegado a sus normativas.

A esta situación le acompañan medias altas para cada uno de los ítems, indicando que se cataloga como siempre el que: se identifican las aéreas de negocio que generan ingresos $o$ beneficios, la variedad de productos que ofrece la banca universal dan origen a nuevas áreas de negocio, la gestión tecnológica le permite a la banca universal captar nuevas áreas de negocio y un portafolio combinado de áreas permitirá mejorar la posición de la banca universal.

Tabla 2. Indicador: Áreas de negocio

\begin{tabular}{|c|c|c|c|}
\hline & ÍTEM & $\overline{\mathbf{X}}$ & CATEGORÍA \\
\hline 7. & $\begin{array}{l}\text { Se identifican las aéreas de negocio que generan ingresos o } \\
\text { beneficios }\end{array}$ & 4,63 & Siempre \\
\hline 8. & Se identifican los beneficios generados en las aéreas de negocio & 4,58 & Siempre \\
\hline 9. & $\begin{array}{l}\text { La variedad de productos que ofrece la banca universal dan origen } \\
\text { a nuevas áreas de negocio }\end{array}$ & 4,52 & Siempre \\
\hline 10. & $\begin{array}{l}\text { La gestión tecnológica le permite a la banca universal captar } \\
\text { nuevas áreas de negocio }\end{array}$ & 4,55 & Siempre \\
\hline \multirow[t]{2}{*}{11.} & $\begin{array}{l}\text { Un portafolio combinado de áreas permitirá mejorar la posición } \\
\text { de la banca universal }\end{array}$ & 4,66 & Siempre \\
\hline & TOTAL INDICADOR: & 4,59 & SIEMPRE \\
\hline
\end{tabular}

Fuente: La investigadora (2020)

Es evidente, de acuerdo a lo mostrado, que el área de negocio, siempre forma parte de los procesos de negocio de la banca universal, como unidades estratégicas del negocio, permitiendo crear una cartera de negocios con la necesidad de un crecimiento rentable para la banca, a fin de valorar y seleccionar oportunidades de mercado y elaborar estrategias para alcanzarlas con su objetivo correspondiente. 
Lo mostrado alcanza a validar, de manera muy alta, lo postulado por Muñiz (2003), para quien el área de negocio es una unidad organizativa que asume la responsabilidad de realizar todas las funciones y trabajo ya que tienen capacidad de decisión y una visión global del funcionamiento de su unidad. La estructura por áreas de negocio es más efectiva cuando los costos por departamento centralizados son muy reducidos.

También se valida el criterio de la investigadora, cuando considera que las áreas de negocio, son segmentos identificables del entorno con el que la banca hace o puede hacer negocio, a fin de responder con su oferta a unas demandas de producto, mercadoclientes, tecnología, geografía diferenciados, actuando competitivamente buscando maximizar ingresosfacturación y resultados-beneficios.
En la tabla 3 se resume el comportamiento del indicador operaciones del negocio, donde se observa que el mismo alcanzó un promedio de 4,62, ubicado en la categoría siempre al considerar los encuestados que la banca universal a través de sus operaciones de negocio pueden generar un gran mercado competitivo apegado a las normativas.

De manera específica, puede observarse como siempre se examinan cada operación de negocio para establecer que actividades generan ingresos, al mismo tiempo se identifican las funciones que aportan resultados al mercado bancario y se realiza periódicamente un diagnostico situacional actual de la banca que permita detectar las operaciones de negocio.

Tabla 3. Indicador: Operaciones de negocio

\begin{tabular}{llcc}
\hline \multicolumn{1}{c}{ ÍTEM } & $\overline{\mathbf{X}}$ & CATEGORÍA \\
\hline 12. & $\begin{array}{l}\text { Se examinan cada operación de negocio para establecer } \\
\text { que actividades generan ingresos. }\end{array}$ & 4,64 & Siempre \\
13. & $\begin{array}{l}\text { Se identifican las funciones que aportan resultados al } \\
\text { mercado bancario. }\end{array}$ & 4,61 & Siempre \\
14. Se realiza periódicamente un diagnostico situacional \\
actual de la banca que permita detectar las operaciones de \\
negocio $\quad$ TOTAL INDICADOR:
\end{tabular}

Fuente: La investigadora (2020)

Esto demarca el que en la banca analizada, las actividades, funciones y tareas se realizan para generar ingresos o aportaciones a los resultados. Lo mostrado, coincide de muy alta manera con lo expuesto por Estupiñan (2006), para quien las operaciones de negocio deben revelar si tienen mecanismo de seguridad óptimos en la negociación, si se hicieron en las condiciones pactadas propias de la entidad que aseguren la comprobación de las condiciones pactadas para evitar suspicacias en el momento de la verificación de las operaciones, indicando además si la contabilización de las operaciones se realiza rápida y precisa para evitar errores que puedan significar perdidas.

Situación que coincide con el criterio de la investigadora, cuando afirma que las operaciones de negocio, es todo lo que pasa dentro de la banca para mantenerla de pie y ganando dinero y una vez que están establecidas las operaciones de negocio y particularmente después del crecimiento, es importante evaluar y analizar periódicamente dichas operaciones para identificar ineficiencias y mejorar la comunicación. 
Luego de analizados los resultados obtenidos para cada uno de los indicadores que conforman la dimensión procesos de negocio, en la tabla 4 se reflejan los resultados alcanzados en dicha dimensión. Observándose un promedio de 4,62 e indicando que siempre se conoce con exactitud lo que establece la banca universal en sus bases del negocio orientados a la producción en el mercado a través de un portfolio de productos y servicios apoyados en la innovación, tecnología y la gestión de las áreas de negocio.

Tabla 4. Procesos de negocio

\begin{tabular}{lcc}
\hline \multicolumn{1}{c}{ INDICADOR } & $\overline{\mathbf{X}}$ & CATEGORÍA \\
\hline Bases del negocio & 4,65 & Siempre \\
Área del negocio & 4,59 & Siempre \\
Operaciones del negocio & 4,62 & Siempre \\
TOTAL & 4,62 & SIEMPRE \\
\hline
\end{tabular}

Fuente: La investigadora (2020)

Con base a lo descrito, puede decirse entonces que los procesos de negocio se describen como aquellos que hacen referencia a los resultados no sólo económicos, sino al cumplimiento de los objetivos que por naturaleza y esenciales características corresponden a la banca universal en Costa Oriental del Lago.

Los resultados validan, en muy alta medida, a Laudon (2004), para quien los procesos de negocios se refieren a la manera especial de organizar, coordinar y enfocar el trabajo para elaborar un producto o servicio valioso. Asimismo, se tiene coincidencia con De Carlos (2004), cuando afirma que el proceso de negocio consiste en la identificación del conjunto de orientaciones, funciones, actividades que tienen directa relación y de los que dependen en forma decisiva, y principal, los resultados económicos en términos de ingresos o las pérdidas o ganancias o el cumplimiento de sus propósitos esenciales.

Esto es coincidente con el criterio de la investigadora, cuando afirma que el proceso de negocio, es una serie de pasos realizados por la banca universal en busca de mejorar la eficiencia y la eficacia por medio de la gestión sistemática de los mismos. Dichos pasos tienen un orden específico de actividades de trabajo, que se realizan en el tiempo, en lugares específicos y por personas o sistemas, con un comienzo, un fin, con entradas y salidas claramente definidas.

\section{CONCLUSIONES}

Durante décadas las empresas y las organizaciones en general han venido evolucionando e introduciendo también cambios en su estructura, en sus procesos, en sus fines, su liderazgo y en su gerencia. Estos cambios son imprescindibles y han sido necesarios para su progresiva adaptación a las modificaciones de su entorno, llámese contexto económico, político, tecnológico, social y de mercado.

Así las cosas, la banca universal no escapa de estas necesidades de cambio ya que de ellos depende la supervivencia de dichas organizaciones en el mercado, la cual estará determinada por la implantación efectiva de los procesos de negocio. Partiendo de estas premisas, en la presente investigación se llegó a las siguientes conclusiones:

Se evidencio que los bancos objeto de estudio siempre utilizan la base del negocio, el área del negocio y las operaciones del negocio como procesos de negocio que manejan recursos financieros. En relación al indicador base del negocio, los resultados permiten inferir que quienes gestionan estos procesos toman decisiones básicas al identificar nuevas oportunidades de crecer a fin de segmentar otras formas de mercados y decidir sobre cuáles son las nuevas tecnologías que deben adoptar.

Respecto al área de negocio, este siempre forma parte de los procesos de negocio de la banca universal, como unidades estratégicas del negocio, 
permitiendo crear una cartera de negocios con la necesidad de un crecimiento rentable para la banca, a fin de valorar y seleccionar oportunidades de mercado y elaborar estrategias para alcanzarlas con su objetivo correspondiente.

Finalmente, se evidenció que las operaciones de negocio, se realizan a través de actividades, funciones y tareas que permiten generar ingresos o aportaciones a los resultados de la banca analizada.

\section{REFERENCIAS BIBLIOGRAFICAS}

Abadal, E. (2001). Sistemas y servicios de información digital. Publicado por Universitat de Barcelona, UnitedStates

Casado, A. y Sellers, R. (2006). Dirección de Marketing: Teoría y Práctica. Editorial: Editorial Club Universitario. Malaga, España

Charan, R. (2007). Know-How. Editorial Norma Bogotá Colombia
De Carlos, C. (2004). Análisis estratégico del negocio. Ediciones universitarias de Val Paraíso. Chile

Estupiñan, R. (2006). Control interno y Fraude. Ediciones Ecoe. Bogotá Colombia

Laudon, J. (2004). Sistema de información gerencial administración de la empresa digital. Pearson Educación, s.a. D.F. México.

Muñiz, R. 2003. Estrategia de marketing: La dirección estratégica. Disponible en http://www.marketing.xxi.com//a_direccion_es trategical6htm

Santos, N. (2001). El negocio bancario. Revista industrial data. Volumen 4 (1) : pág. 25-33. Disponible en: http://sisbib.unmsm.edu.pe/bibvirtual/publicac iones/indata/v04_nl/negocio.htm \#arriba

Sommerville, I. (2005). Ingeniería del Software. Editorial Pearson Educación, SA Madrid España 